\title{
OPEN Identification of the first highly selective inhibitor of human lactate dehydrogenase B
}

\author{
Sachio Shibata ${ }^{1,4 凶}$, Satoshi Sogabe ${ }^{1,4}$, Masanori Miwa ${ }^{1}$, Takuya Fujimoto ${ }^{2}$, \\ Nobuyuki Takakura ${ }^{2}$, Akihiko Naotsuka ${ }^{1}$, Shuji Kitamura ${ }^{2}$, Tomohiro Kawamoto ${ }^{1 \bowtie}$ \& \\ Tomoyoshi Soga ${ }^{3}$
}

Lactate dehydrogenase (LDH) catalyses the conversion of pyruvate to lactate and NADH to NAD ${ }^{+}$it has two isoforms, LDHA and LDHB. LDHA is a promising target for cancer therapy, whereas LDHB is necessary for basal autophagy and cancer cell proliferation in oxidative and glycolytic cancer cells. To the best of our knowledge, selective inhibitors for LDHB have not yet been reported. Here, we developed a high-throughput mass spectrometry screening system using an LDHB enzyme assay by detecting NADH and $\mathrm{NAD}^{+}$. As a result, we identified a small-molecule LDHB selective inhibitor AXKO0046, an indole derivative. This compound exhibited uncompetitive $L D H B$ inhibition $\left(E C_{50}=42 \mathrm{nM}\right)$. X-ray crystallography revealed that AXKO-0046 bound to the potential allosteric site away from the LDHB catalytic active site, suggesting that targeting the tetramerisation interface of the two dimers is critical for the enzymatic activity. AXKO-0046 and its derivatives can be used to validate LDHBassociated pathways in cancer metabolism.

Cancer cells can reprogram various genes to promote their rapid proliferation and metastatic potential ${ }^{1}$. Unlike most normal cells, cancer cells can adapt to various microenvironments, such as hypoxia, glucose and other nutrient deficiencies, and acidosis ${ }^{1}$. Moreover, in tumours and other proliferating or developing cells, a metabolic switch from normal oxidative phosphorylation to aerobic glycolysis is common ${ }^{1}$. This adaptation, known as the Warburg effect, allows cancer cells to produce ATP from glucose by promoting glycolysis to produce lactate from the mitochondrial pyruvate pool, even in the presence of oxygen ${ }^{1,2}$. Increased aerobic glycolysis provides cancer cells with a growth advantage, despite its energetic inefficiency compared with oxidative phosphorylation ${ }^{3}$.

During aerobic glycolysis, the conversion of pyruvate to lactate is mediated by cytosolic lactate dehydrogenase (LDH) enzymes with nicotinamide adenine dinucleotide (NADH) as a cofactor, which is converted to nicotinamide adenine dinucleotide $\left(\mathrm{NAD}^{+}\right)$. The $\mathrm{LDH}$ enzymes are tetrameric enzymes comprising two separate subunits, $\mathrm{M}$ and $\mathrm{H}$, and can form five isozymes, namely, the LDH1 $(4 \mathrm{H}), \mathrm{LDH} 2(3 \mathrm{H}, 1 \mathrm{M}), \mathrm{LDH} 3(2 \mathrm{H}, 2 \mathrm{M}), \mathrm{LDH} 4(1 \mathrm{H}$, $3 \mathrm{M})$, and LDH5 (4M) subunits. LDH1 and LDH5 are transcribed from the LDHB and LDHA genes, respectively ${ }^{4}$.

Previous studies have reported that LDHA (LDH5) plays a critical role in cancer. For example, in patients with gastric cancer or non-small-cell lung cancer, high LDHA levels are correlated with tumour size and poor prognosis ${ }^{5,6}$. In addition, LDHA silencing by small interfering RNA or short hairpin RNA inhibits cell growth and tumorigenic potential both in vitro and in xenograft models $s^{7,8}$. Therefore, several LDHA inhibitors have been developed whose hit target compounds have been identified through high-throughput screening (HTS $)^{9}$.

LDHB (LDH1) is associated with aggressive cancer phenotypes ${ }^{10,11}$. One study used clinical samples derived from patients with colorectal cancer and found that MYC expression is highly correlated with the expression of various metabolic genes. In that study, 231 unique metabolic genes were identified, and the LDHB levels were upregulated, whereas the LDHA levels remained unchanged in colorectal cancer ${ }^{12}$. Furthermore, LDHB is a key contributor to lysosomal activity and autophagy in cancer ${ }^{13}$. Because various cancer cells upregulate autophagy, which is required to support metabolism, tumourigenesis, and resistance to therapy ${ }^{14}$, inhibition of LDHB could be an excellent target for the prevention and treatment of several cancers. Moreover, LDHB is differentially expressed in triple-negative breast cancers, defined by the absence of detectable estrogen receptor and progesterone receptor expression and the lack of human epidermal growth factor 2 gene amplification ${ }^{11}$.

${ }^{1}$ Discovery Biology, Discovery Science, Axcelead Drug Discovery Partners, Inc., 2-26-1 Muraoka-Higashi, Fujisawa, Kanagawa, Japan. ${ }^{2}$ Chemistry, Discovery Science, Axcelead Drug Discovery Partners, Inc., 2-26-1 Muraoka-Higashi, Fujisawa, Kanagawa, Japan. ${ }^{3}$ Institute for Advanced Biosciences, Keio University, 246-2 Mizukami, Kakuganji, Tsuruoka, Yamagata, Japan. ${ }^{4}$ These authors contributed equally: Sachio Shibata and Satoshi Sogabe. 
a<smiles>[R]N1C=CC([2H])C(C(N)=O)=C1</smiles>

$\mathrm{Q} 1 / \mathrm{Q} 3=664.2 / 397.0$

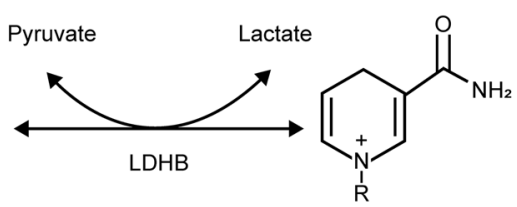

$\mathrm{Q} 1 / \mathrm{Q} 3=662.1 / 540.1$

C

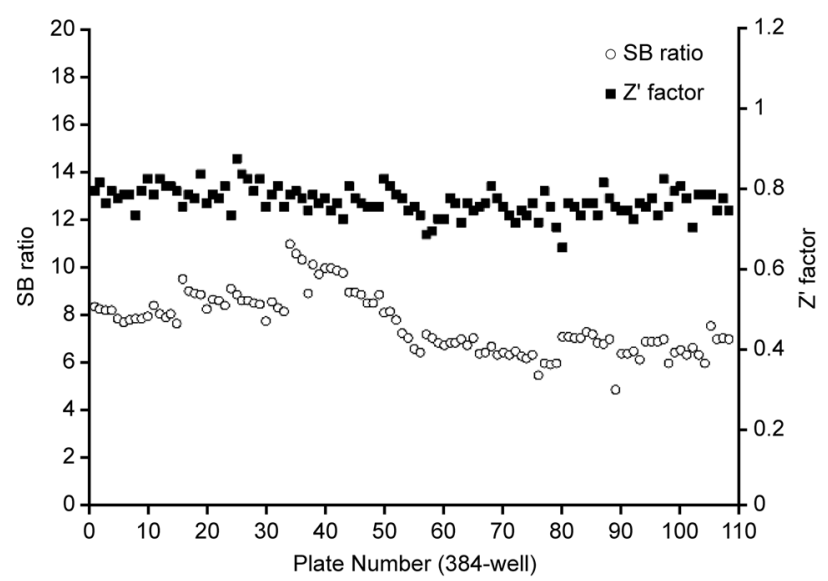

Primary screen using RapidFire MS

37,122-well (10 mixed compounds/well)

Criterion: $30 \%$ or more inhibition at $10 \mu \mathrm{M}$ compound concentrations

Selected 81 -well hits $(0.22 \%$ hit rate) for deconvolution assay

Deconvolution assay and Purity check

Criterion: $30 \%$ or more inhibition at $30 \mu \mathrm{M}$ compound concentrations Criterion for Purity: more than $80 \%$

Selected 22 compounds $(0.006 \%$ hit rate $)$

Selected 22 compounds $(0.006 \%$ hit rate)

Selectivity test for LDHB/LDHA

Assay dose: $3,10,30,100$ and $300 \mu \mathrm{M}(\mathrm{n}=2)$

Selected the indole series as hit chemotype

ב.

Evalution of related compounds on hit chemotype

Assay dose: $0.01,0.1,1,10$ and $100 \mu \mathrm{M}(\mathrm{n}=2)$

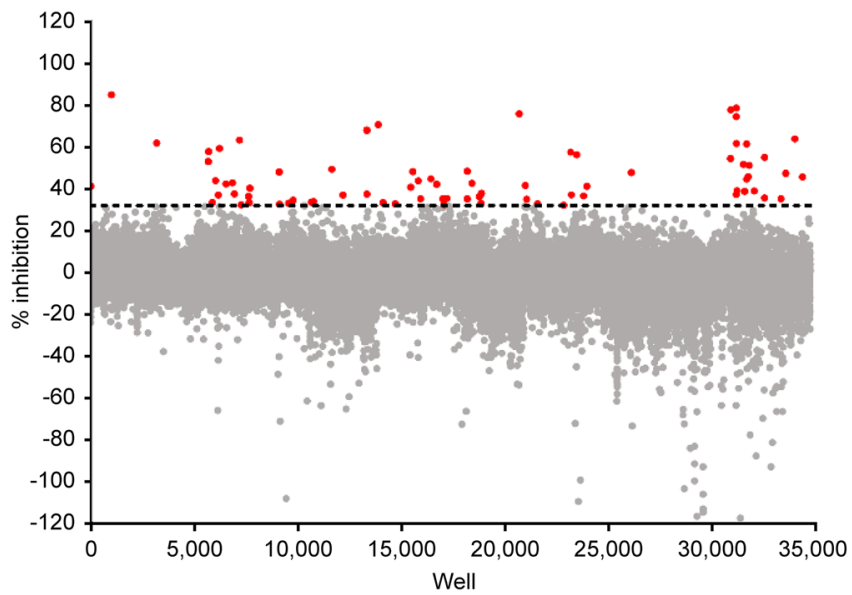

Figure 1. Primary screening for inhibitors of lactate dehydrogenase B (LDHB). (a) LDHB-catalysed reaction scheme. $\mathrm{NADH}$ and $\mathrm{NAD}^{+}$were detected using mass spectrometry, and their respective MRMs are shown. (b) Screening cascade and the results of primary screening. The high-throughput screening cascade consisted of the primary screening, profiling assay, hit validation, and characterisation. A diverse library of small compounds from Axcelead at $10 \mu \mathrm{M}$ was screened against LDHB. (c) SB ratio (open circles) and Z'-factor values (filled squares) of the overall primary screening. (d) Scatterplots of primary screening data and selection of hit wells exhibiting greater than $30 \%$ inhibitory activity (red circles).

$L D H B$ knockdown selectively reduces the proliferation of breast cancer cells, both in vitro and in vivo ${ }^{11}$. Triplenegative breast cancer cells exhibit enhanced glycolysis and utilise pyruvate or lactate as an energy source ${ }^{11}$. Therefore, we hypothesised that the inhibition of LDHB may be a promising therapeutic approach for targeting cancer metabolism.

Despite the suggested association between LDHB and cancer metabolism, LDHB-selective inhibitors remain unexplored. In this study, to identify selective LDHB inhibitors, we developed a screening system to monitor the conversion of NADH to $\mathrm{NAD}^{+}$by $\mathrm{LDHB}$ using the RapidFire-Mass (RF-MS) system. This assay system was robust and avoided false-positive results. We then identified that AXKO-0046, an indole derivative, selectively inhibited $\mathrm{LDHB}$ activity in an uncompetitive manner with respect to $\mathrm{NADH}$ and pyruvate. Moreover, structural analysis showed that AXKO-0046 did not bind in the catalytic site of the enzyme but significantly bound to LDHB in the novel allosteric site of the tetramer. The allosteric site was unique to LDHB based on sequence alignment, resulting in LDHB-selective inhibition. Overall, our findings suggest that AXKO-0046 may be a promising chemical probe to elucidate the role of LDHB-associated pathways in cancer metabolism.

\section{Results}

Development and optimisation of RF-MS for LDHB activity. To identify small-molecule LDHB inhibitors, we developed an assay system in a 384-well format to measure LDHB activity through NADH and $\mathrm{NAD}^{+}$by RF-MS (Fig. 1a). The integrated peak areas were linear for concentrations of the NADH or NAD ${ }^{+}$ standard between 1 and $100 \mu \mathrm{M}$ (Supplementary Fig. 1). The extracted ion chromatogram of NADH also showed a $\mathrm{NAD}^{+}$fragment originating from the in-source decay (ISD) of NADH during ionisation. Because the ISD of 
a

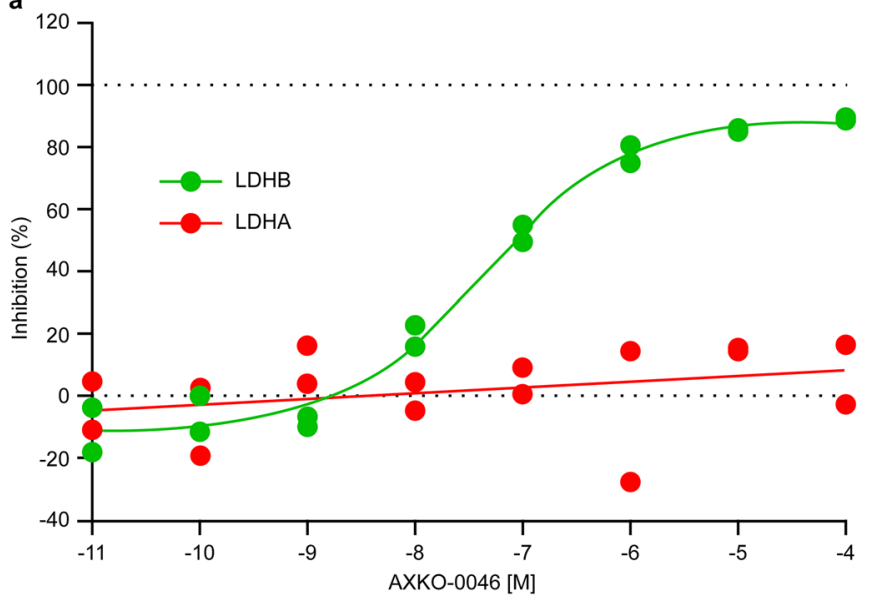

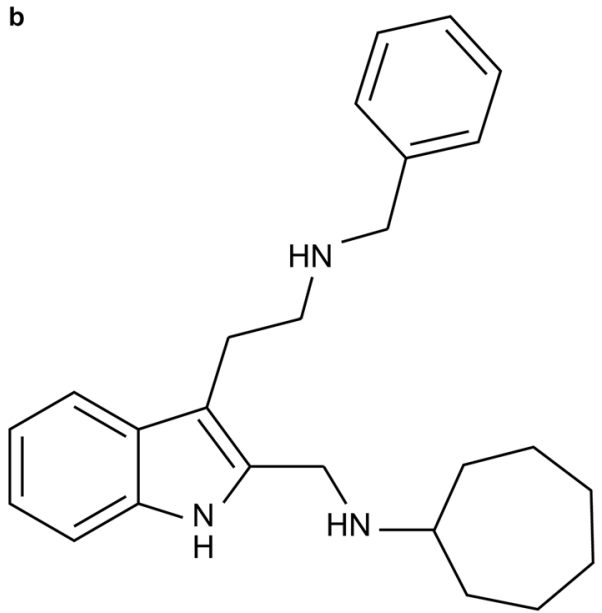

Figure 2. Concentration response curve and the structure of AXKO-0046. (a) Concentration response curves and $\mathrm{EC}_{50}$ values of AXKO-0046. AXKO-0046 selectively inhibited LDHB (green circles) but did not inhibit LDHA (red circles). The $\mathrm{EC}_{50}$ for LDHB was $42 \mathrm{nM}$. The curves were fitted to the standard four-parameter logistic equation. (b) Structure of AXKO-0046.

$\mathrm{NAD}^{+}$observed with $\mathrm{NADH}$ was less than $10 \%$ of the peak area of $\mathrm{NADH}$, there was no contribution to the detection of LDHB activity.

Next, we optimised the assay conditions of RF-MS. Titration of human LDHB was examined at concentrations ranging from 0.0625 to $1 \mathrm{nM}$. Supplementary Fig. 2 shows a linear relationship between the velocity and enzyme concentration $(0.0625-0.5 \mathrm{nM})$. We used $0.25 \mathrm{nM}$ LDHB throughout assay development and screening to ensure enzyme activation. Next, the concentrations of NADH and pyruvate were optimised. The $\mathrm{K}_{\mathrm{m}}$ values of NADH and pyruvate were 64 and $116 \mu \mathrm{M}$, respectively, in the presence of $500 \mu \mathrm{M}$ pyruvate and increasing concentrations of NADH or $500 \mu \mathrm{M}$ NADH and increasing concentrations of pyruvate (Supplementary Fig. 2). The $\mathrm{K}_{\mathrm{m}}$ values obtained from the two assays were consistent with the reported values for LDHB ${ }^{15,16}$. Based on the $\mathrm{K}_{\mathrm{m}}$ of each substrate and the robustness of the assay signal, NADH and pyruvate concentrations were both set at $75 \mu \mathrm{M}$ for screening.

HTS of LDHB inhibitors using RF-MS. The screening campaign was performed on an in-house pooled diverse library of more than 345,000 compounds at a concentration of $10 \mu \mathrm{M}$, as shown in the workflow (Fig. 1b). Assay quality was acceptable because Z' factors exceeded 0.65 and a signal-to-noise ratio greater than 5.0 was obtained (Fig. 1c). The hit rate was $0.22 \%$ when the threshold was set at greater than or equal to $30 \%$ of inhibition compared with the control (Fig. 1d).

To identify the hit compounds in pooled samples, deconvolution assays were conducted at a compound concentration of $30 \mu \mathrm{M}$. Compounds containing oxalate salt, which had inhibitory activities toward LDH, were excluded as undesirable inhibitors because oxalate and its derivatives show only moderate potency and selectivity ${ }^{17,18}$. Thus, 22 compounds showed activity (greater than or equal to $30 \%$ inhibition rate and greater than or equal to $80 \%$ purity), corresponding to a hit rate of $0.006 \%$. Subsequent dose-response experiments determined the $\mathrm{EC}_{50}$ values for all identified hit compounds.

Comparison of the $\mathrm{EC}_{50}$ Values of hit compounds for LDHB and LDHA. To compare the selectivity potency of the 22 selected compounds, we evaluated the compounds against human LDHB and LDHA by RF-MS. To obtain comparable $\mathrm{EC}_{50}$ values, the substrate concentration was fixed to each $\mathrm{K}_{\mathrm{m}}$ determined using our assay conditions for each substrate pair. For LDHA, time-dependent accumulation of NADH was confirmed in a protein concentration-dependent manner (Supplementary Fig. 2). The $\mathrm{K}_{\mathrm{m}}$ value was also determined for the LDHA reaction. All EC $\mathrm{E}_{50}$ values are listed in Supplementary Table 1.

Four compounds (i.e., AXKO-0004, AXKO-0008, AXKO-0010, and AXKO-0013) exhibited significant differences in potency $\left(\mathrm{EC}_{50}\right)$ between LDHB and LDHA. Although AXKO-010 was a partial inhibitor at the highest compound concentration, this was not due to compound concentration effects, such as aggregation, instability, and assay interference. Moreover, this compound exhibited LDHB inhibitory activity, with an $\mathrm{EC}_{50}$ of less than or equal to $3 \mu \mathrm{M}$, but no inhibitory activity against LDHA at $300 \mu \mathrm{M}$, thus showing greater than 100 -fold selectivity over LDHA (Supplementary Table 1).

To identify more potent compounds, we next assessed the inhibitory effects of 75 indole derivatives with substructural similarity from the compound library. AXKO-0046 was identified as N-(\{3-[2-(benzylamino) ethyl]$1 \mathrm{H}$-indol-2-yl $\}$ methyl) cycloheptanamine and achieved highly potent LDHB inhibitory activity $\left(\mathrm{EC}_{50}=42 \mathrm{nM}\right)$ with selectivity over LDHA (Fig. 2).

Characterisation of AXKO-0046. To elucidate the underlying inhibitory mechanism, substrate competition assays were performed. LDHB inhibitory activity for AXKO-0046 was assessed at different concentrations 

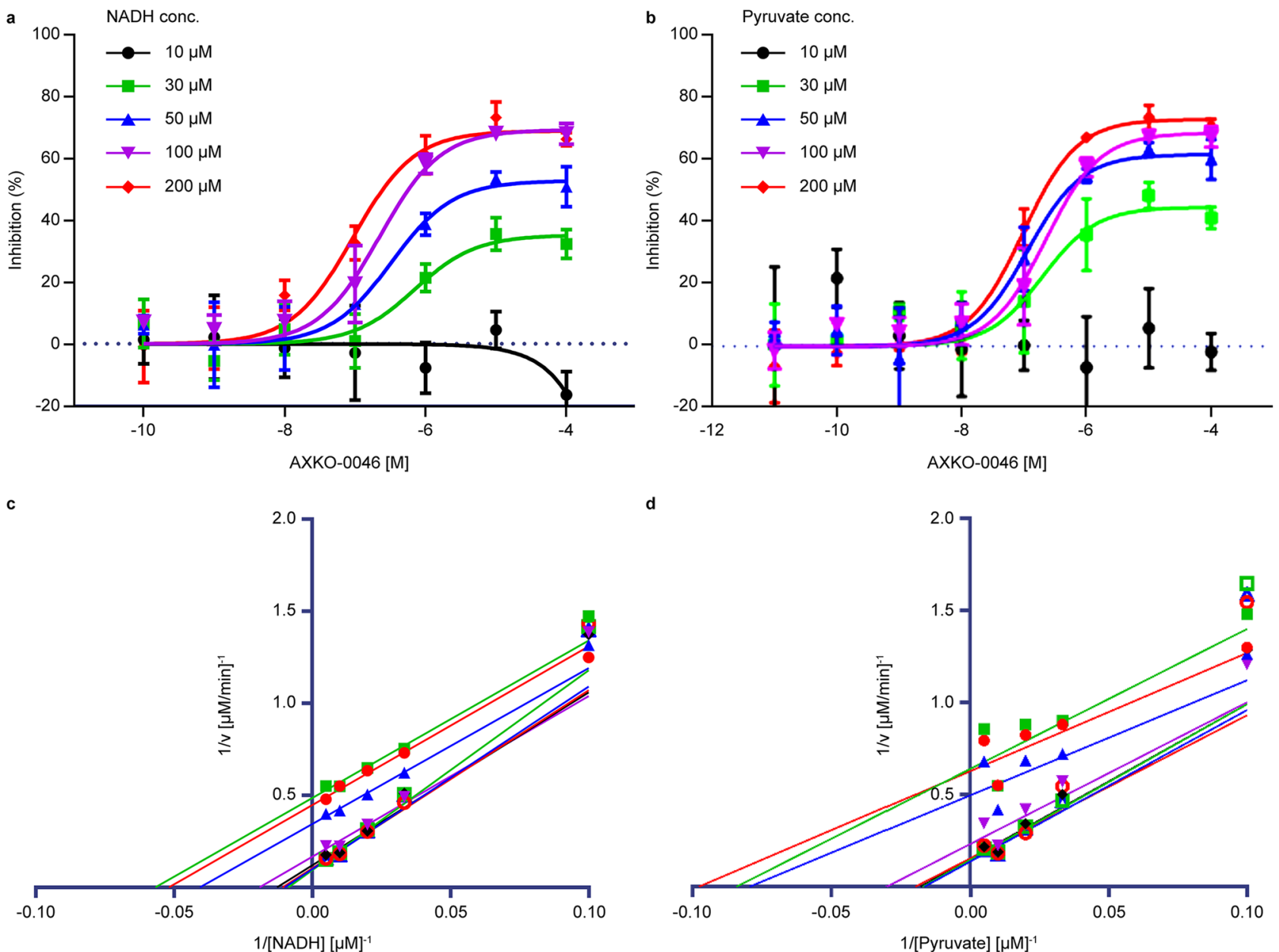

d
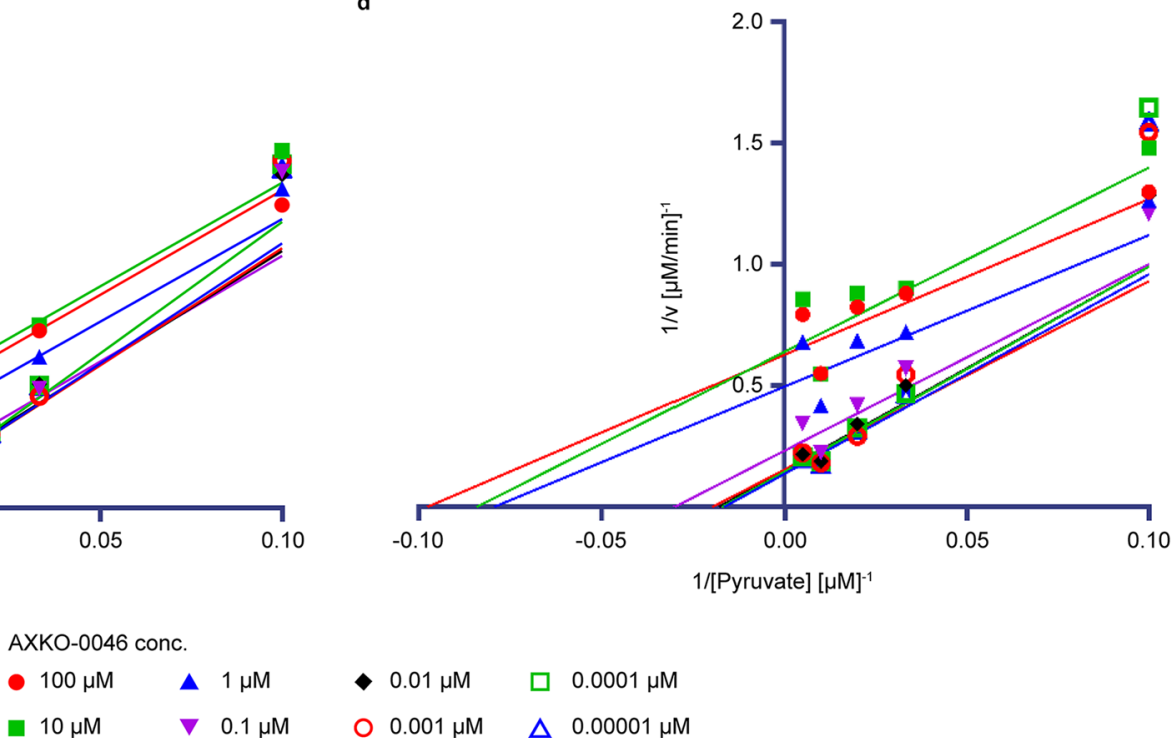

$\begin{array}{ll}\bullet & 0.01 \mu \mathrm{M} \\ 0 & 0.001 \mu \mathrm{M}\end{array}$

$\square 0.0001 \mu \mathrm{M}$

$\Delta 0.00001 \mu \mathrm{M}$

Figure 3. Biochemical characterisation of AXKO-0046. (a,b) LDHB inhibition by AXKO0046 was studied using varying concentrations of (a) NADH and (b) pyruvate. The $\mathrm{EC}_{50}$ values of $\mathrm{AXKO}-0046$ decreased with increasing pyruvate and NADH concentrations. Data shown are mean \pm standard deviation $(n=3)$. $(\mathbf{c}, \mathbf{d})$ Lineweaver-Burk plots of the kinetic data in Table 1 with nonlinear regression analysis of (c) NADH and (d) pyruvate.

of the substrate. The inhibitory activity of AXKO-0046 increased depending on the concentration of NADH. Similar to NADH, AXKO-0046 exhibited decreasing EC Fn $_{5}$ values at increasing pyruvate concentrations, indicating that it was uncompetitive with both substrates (Fig. 3a,b, and Supplementary Table 2).

Moreover, the $\mathrm{K}_{\mathrm{m}}$ and $\mathrm{V}_{\max }$ values for LDHB were determined in the presence AXKO-0046. As the AXKO0046 concentration increased in the reaction, both $\mathrm{V}_{\max }$ and $\mathrm{K}_{\mathrm{m}}$ decreased proportionally (Supplementary Fig. 3 and Table 1). The Lineweaver-Burke plot lines were nearly parallel (Fig. 3c,d), indicating uncompetitive inhibition with a strong preference for inhibiting the enzyme-substrate complex.

We then assessed whether there was any time dependence to the onset of inhibition by varying the time for which AXKO-0046 and LDHB were pre-incubated before initiating the enzymatic reaction. Following preincubation with LDHB for $120 \mathrm{~min}$, the inhibition potential of AXKO-0046 did not increase with pre-incubation time (Supplementary Fig. 4).

Characterisation of AXKO-0046 derivatives. To assess the structure-activity relationships (SARs) of AXKO-0046, its derivatives were synthesised, and their inhibitory activities were evaluated. Methyl scanning is a well-known systematic approach to determine interaction sites between small molecules and macromolecular targets, thus providing opportunities for structural modifications to improve potency ${ }^{19}$. The structure and inhibitory activity of each compound are listed in Fig. 4.

AXKO-0046 contains three hydrogen bond donors, that is, the NH group of indole, benzylamine, and cycloheptylamine, which can interact with the counter amino acid residues of LDHB. The introduction of a methyl group to the benzylamino group (AXKO-0060) resulted in a moderate loss of inhibitory activity $\left(\mathrm{EC}_{50}=0.25 \mu \mathrm{M}\right)$, 


\begin{tabular}{|c|c|c|c|c|c|c|c|c|}
\hline & \multicolumn{8}{|c|}{ AXKO-0046 concentration $[\mu \mathrm{M}]$} \\
\hline & 0.00001 & 0.0001 & 0.001 & 0.01 & 0.1 & 1 & 10 & 100 \\
\hline \multicolumn{9}{|l|}{ NADH } \\
\hline $\mathrm{V}_{\max }(\mu \mathrm{M} / \mathrm{min})$ & 10.1 & 10.7 & 9.7 & 8.3 & 6.0 & 2.9 & 2.1 & 2.2 \\
\hline $\mathrm{SD} \pm$ & 0.1 & 0.8 & 0.8 & 0.7 & 0.5 & 0.1 & 0.1 & 0.1 \\
\hline $\mathrm{K}_{\mathrm{m}}(\mu \mathrm{M})$ & 100.4 & 116 & 93.5 & 78.4 & 52.5 & 24.6 & 17.6 & 19.3 \\
\hline $\mathrm{SD} \pm$ & 20.5 & 17.4 & 15.7 & 15.3 & 10.7 & 2.3 & 2.1 & 3.0 \\
\hline \multicolumn{9}{|l|}{ Pyruvate } \\
\hline $\mathrm{V}_{\max }(\mu \mathrm{M} / \mathrm{min})$ & 7.3 & 6.9 & 6.5 & 6.6 & 4.3 & 2.0 & 1.6 & 1.6 \\
\hline $\mathrm{SD} \pm$ & 0.9 & 0.7 & 0.9 & 0.8 & 0.7 & 0.2 & 0.1 & 0.1 \\
\hline $\mathrm{K}_{\mathrm{m}}(\mu \mathrm{M})$ & 60.3 & 58.3 & 50.5 & 55.2 & 33.2 & 12.6 & \begin{tabular}{|l|}
11.9 \\
\end{tabular} & 10.2 \\
\hline $\mathrm{SD} \pm$ & 19.1 & \begin{tabular}{|l|}
15.7 \\
\end{tabular} & \begin{tabular}{|l|l|}
18.8 \\
\end{tabular} & \begin{tabular}{|l|}
16.6 \\
\end{tabular} & \begin{tabular}{|l|l}
16.3 \\
\end{tabular} & 6.4 & 5.3 & 4.3 \\
\hline
\end{tabular}

Table 1. Effects of AXKO-0046 on $\mathrm{K}_{\mathrm{m}}$ and $\mathrm{V}_{\max }$ of $\mathrm{NADH}$ and pyruvate on LDHB enzyme activity. Data shown are mean \pm standard deviation $(\mathrm{n}=3)$.

\begin{tabular}{|c|c|c|c|c|}
\hline Compound & $\mathbf{R}^{1}$ & $\mathbf{R}^{2}$ & $\mathbf{R}^{3}$ & $\mathrm{EC}_{50}(\boldsymbol{\mu M})$ \\
\hline AXKO-0046 & $\mathrm{H}$ & $\mathrm{H}$ & $\mathrm{H}$ & $>042$ \\
\hline AXKO-0067 & $\mathrm{Me}$ & $\mathrm{H}$ & $\mathrm{H}$ & 0.25 \\
\hline AXKO-0060 & $\mathrm{H}$ & $\mathrm{Me}$ & $\mathrm{H}$ & 7.4 \\
\hline AXKO-0077 & $\mathrm{H}$ & $\mathrm{H}$ & $\mathrm{Me}$ & 8.3 \\
\hline AXKO-0058 & $\mathrm{H}$ & $\mathrm{Me}$ & $\mathrm{Me}$ & 8.2 \\
\hline
\end{tabular}

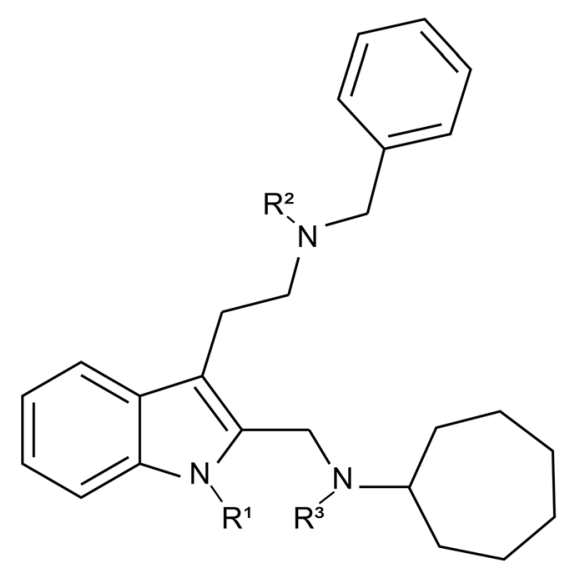

Figure 4. Chemical structure of $\mathrm{AXKO}-0046$ derivatives and their $\mathrm{EC}_{50}$ values.

whereas the addition of the methyl group at the cycloheptylamino group (AXKO-0077) resulted in a considerable loss of activity $\left(\mathrm{EC}_{50}=7.4 \mu \mathrm{M}\right)$. The incorporation of two methyl groups into both amino groups (AXKO-0058) simultaneously reduced activity $\left(\mathrm{EC}_{50}=8.3 \mu \mathrm{M}\right)$. Finally, methylation at the 1-position of indole $(\mathrm{AXKO}-0067)$ led to a significant loss of potency $\left(\mathrm{EC}_{50}>100 \mu \mathrm{M}\right)$.

Structural analysis of AXKO-0046 with LDHB. To investigate the binding site, we solved the two crystal structures of $\mathrm{LDHB}$, that is, the binary complex with the cofactor $\mathrm{NADH}$ and the quaternary complex with NADH using the substrate analogue oxamate and the inhibitor AXKO-0046 at 1.80 and $1.55 \AA$ resolution, respectively (Fig. 5a and Supplementary Table 3). Both structures exhibited $\alpha / \beta$ protein folding conserved in the LDHA and LDHB structures, as previously reported ${ }^{20}$. There were two tetramers for the binary complex and one tetramer for the quaternary complex in the asymmetric unit. The average root-mean-square deviation (RMSD) values between the monomers in the asymmetric unit were 0.5 and $0.3 \AA$ for two tetramers of the binary complex and one tetramer of the quaternary complex, respectively. Electron density was visible for NADH and oxamate at the cofactor- and substrate-binding sites, respectively, whereas relatively weak but distinct electron density was visible for AXKO-0046 at the interface between the two monomers (Supplementary Fig. 5). These results 
a

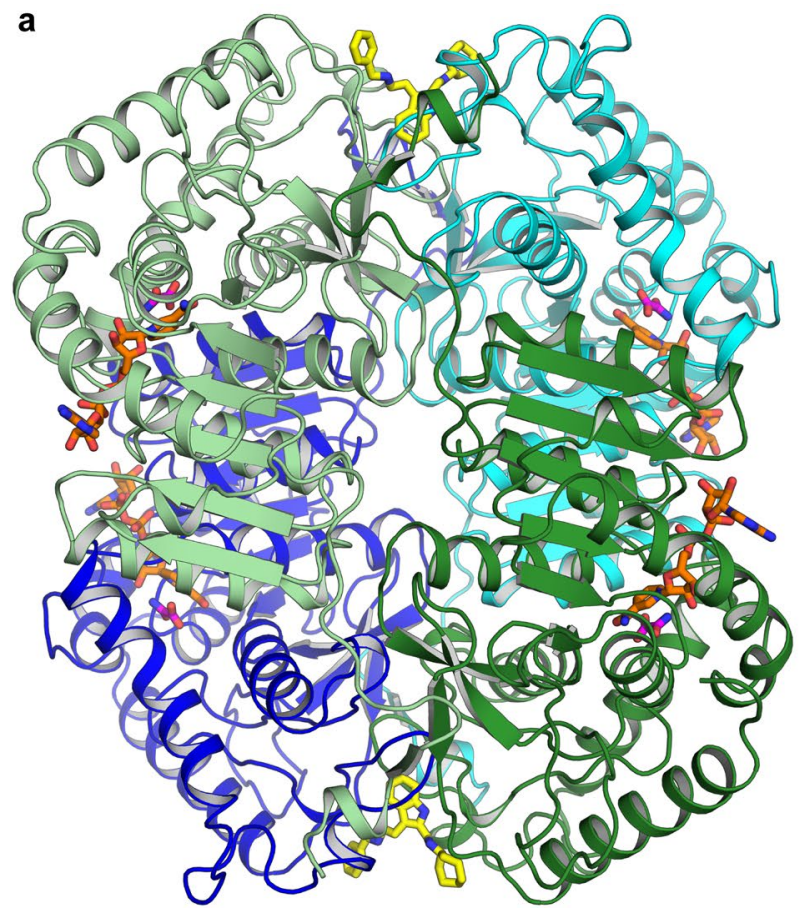

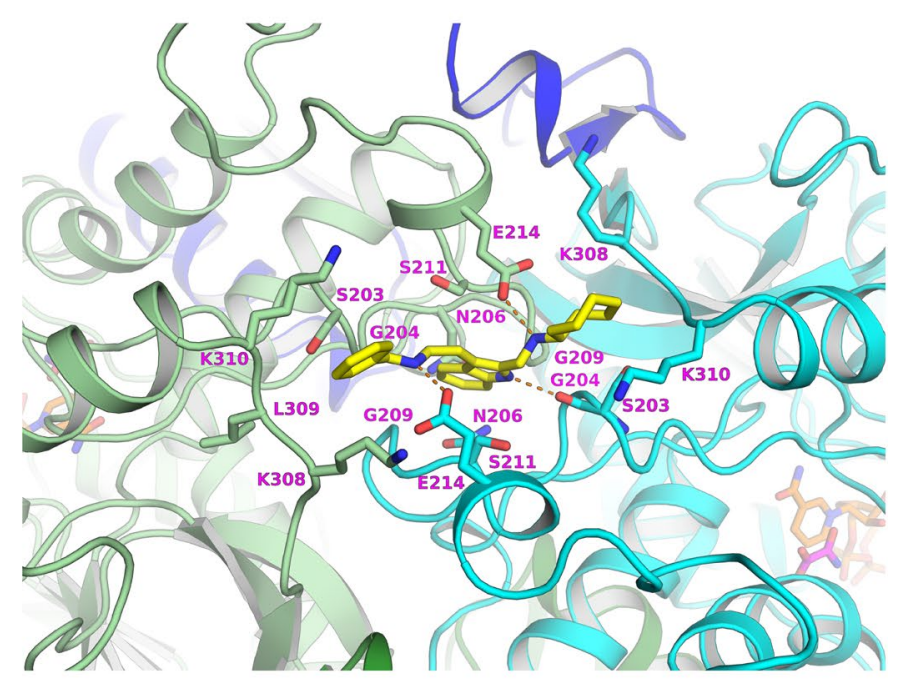

Figure 5. Crystal structure of the quaternary LDHB/NADH/oxamate/AXKO-0046 complex. (a) Ribbon diagram. (b) Close-up view of the binding site of AXKO-0046. Each monomer of the tetramers is shown in lime, forest, cyan, and blue. NADH, oxamate, and AXKO-0046 are depicted as stick models in orange, magenta, and yellow, respectively.

indicated that the compound had alternative conformations on the two-fold axis of the tetramer with relatively low occupancies.

The binding conformation of NADH with the enzyme was essentially identical between the binary complex and the quaternary complex, and their binding interactions were consistent with those of published structures $^{20-22}$. In the quaternary complex, oxamate was bound near the NADH nicotinamide moiety and interacted with the active-site loop (residues Glu101-Leu110), which was in a closed conformation. In contrast, in the binary complex, the active-site loops were partially disordered with an open conformation for the two tetramers in the asymmetric units, except for two of eight monomers in which the loop was well ordered owing to crystal packing (Supplementary Fig. 6). The superimposition of our two structures clearly indicated structural changes corresponding to AXKO-0046 inhibition. Significant structural differences were observed around the active-site loop, with a maximum RMSD of approximately $7 \AA$ for the main-chain atoms. Other marked structural deviations, except for the active site, were observed in the two helices near the active site (residues Trp228-Lys246 and residues Asp311-Asp330), with a maximum RMSD of approximately $2 \AA$ for backbone atoms (Supplementary Fig. 7). These structural variations are further described in the Discussion to elucidate the inhibitory mechanism of AXKO-0046.

AXKO-0046 was located in an interfacial allosteric site between two monomers, approximately more than $20 \AA$ away from the active site (Fig. 5b). Although the compound occupied the site with half-occupancy in the two possible orientations on a two-fold axis, only one orientation was defined owing to the relatively poor electron density, as described in Supplementary Fig. 5. The indole ring thus occupied the small cavity composed of Gly204, Asn206, Gly209, and Ser211. In addition, the NH group of indole formed a hydrogen bond with the main-chain oxygen of Ser203. Two NH groups of benzylamine and cycloheptylamine interacted with the sidechain oxygens of two Glu214 residues. The benzyl and cycloheptyl groups were exposed to the solvent region and surrounded by Lys308 and Lys310, followed by the C-terminal helix with significant structural rearrangements, as described below.

\section{Discussion}

Several metabolic enzymes play major roles in cancer survival; however, they are not extensively exploited as drug targets. Therefore, in this study, we utilised LDHB as a drug target and developed an assay for high-throughput compound screening to identify LDHB inhibitors. To the best of our knowledge, LDHB-specific inhibitors have not been reported owing to the high structural homology between LDHB and LDHA and the high structural homology of their catalytic sites ( $89 \%$ according to the NCBI Basic Local Alignment Search Tool [BLAST] ${ }^{23}$. Furthermore, high-throughput screening for the identification of LDHB-specific inhibitors has not yet been reported.

The metabolic model of lactate shuttling in the tumour microenvironment is known as metabolic symbiosis in tumours $^{24,25}$, where LDHB is a key molecule of the oxidative pathway of lactate that controls metabolic symbiosis between glycolytic and oxidative cancer cells ${ }^{26,27}$. In oxidative cancer cells, lactate is imported by MCT1, located in the cell plasma membrane, and LDHB oxidizes lactate to pyruvate to produce energy. Comparatively, glycolytic 
cancer cells generate lactate from pyruvate via the LDHA reaction. LDHB is needed to sustain glycolytic cancer cell survival. In other words, targeting lactate in oxidative cancer cells could offer a unique opportunity to induce necrosis of distant glycolytic cancer cells known to be resistant to conventional antitumor treatments ${ }^{26}$. Therapeutic strategies to induce necrosis in glycolytic cancer via the inhibition of lactate transport are currently being applied in early clinical trials for cancer treatments using the MCT-1-selective inhibitor AZD3965 ${ }^{28}$. AZD3965 is in the early phase clinical trials (www.clinicaltrials.gov) with phase I expansion cohort enrichment for Burkitt lymphoma and diffuse large B cell lymphoma cases. Moreover, studies have indicated that complete hereditary deficiency of LDHB has no symptomatic consequences in humans ${ }^{29,30}$. On the contrary, patients with hereditary LDHA deficiency have been reported to present with exertional myopathy, erythematosquamous skin lesions, and uterine stiffness during pregnancy ${ }^{31,32}$. Therefore, these results indicate the possibility of the future clinical development of pharmacological inhibitors of LDHB with high efficacy and safety.

To date, in vitro LDH assays have been used to measure the fluorescence of NADH, with a characteristic excitation maximum at $340 \mathrm{~nm}$ and an emission maximum at $480 \mathrm{~nm}^{32,33}$. However, this procedure can give rise to false positives and negatives because of fluorescence interference at the excitation and emission wavelengths of NADH. Although other methods, such as measuring NADH through the conversion of resazurin to resorufin by diaphorase using enzyme-coupled detection methods ${ }^{9}$, have also been suggested, inhibition of the coupling enzymes by test compounds may affect the assay and lead to false positives in HTS campaigns.

Therefore, the Genentech group developed a label-free assay to monitor the conversion of pyruvate to lactate by LDHA using RF-MS ${ }^{34}$. However, this approach was only used for secondary assays to confirm the selected compounds from initial screening employing fluorescence assay. In this study, instead of measuring the conversion of pyruvate to lactate, we established a robust RF-MS assay system to monitor that from NADH to NAD ${ }^{+}$ using LDH activities. This method may be useful not only for LDH but also for other dehydrogenases utilizing $\mathrm{NADH}$ or $\mathrm{NAD}^{+}$. Additionally, this approach allowed us to conduct HTS campaign using a large compound library. The assay showed excellent performance, with an average Z' factor greater than or equal to 0.65 when more than 345,000 compounds were screened.

The hit rate in the screening campaign was very low $(0.006 \%$ with $30 \%$ inhibition threshold). This low hit rate corresponded to the data when screened against LDHA using a small compound library ${ }^{34}$. LDHA is considered a highly intractable target against small molecules ${ }^{18}$. However, we found 22 substantially potent LDHB inhibitors, of which only four compounds (i.e., AXKO-0004, AXKO-0008, AXKO-0010, and AXKO-0013) showed high selectivity for LDHB after retesting at five concentrations. Among these hit compounds, AXKO-0010, which contained a central indole scaffold, showed excellent potency and selectivity as a LDHB inhibitor. However, this compound did not completely inhibit enzyme activity, even after an increase in the concentration. Therefore, we next assessed the inhibitory effects of a subfamily of structurally related compounds to AXKO-0010. We found that 63 of 75 related compounds showed highly potent LDHB inhibitory activities while maintaining selectivity. Of these, AXKO-0046 showed the greatest potency for LDHB inhibition $\left(\mathrm{EC}_{50}=42 \mathrm{nM}\right)$.

Next, the mechanism of LDHB inhibition of AXKO-0046 was evaluated using substrate-competition assays. AXKO-0046 was tested at five different NADH and pyruvate concentrations. Unexpectedly, increasing the concentration of pyruvate and NADH correlated with increased AXKO-0046 inhibitory activity, suggesting an uncompetitive inhibitory mechanism with respect to both NADH and pyruvate. These findings indicated that this compound likely bound to the enzyme-substrate complex. Several other selective LDHA inhibitors, such as galloflavin, oxamate, diacid-malonate scaffolds, and quinoline 3-sulphonamides, have been described; however, these compounds are substrate-competitive inhibitors owing to the interaction with the catalytic site ${ }^{9,35-37}$.

Notably, the NADH concentration in cancer cells is generally elevated and has been reported to range from 168 to $870 \mu \mathrm{M}^{38}$. LDHA likely binds to NADH in cells. Thus, a competitive inhibitor of NADH may yield poor cellular activity ${ }^{37}$. Because AXKO-0046 is uncompetitive with respect to both NADH and pyruvate, we hypothesise that increased intracellular NADH and pyruvate concentrations may increase enzyme inhibition, unlike competitive behaviour. Structural studies provide further insights into the binding site of AXKO-0046 and the interaction residues of the LDHB protein for compound design to inhibit LDHB activity.

The crystal structure of the quaternary complex of LDHB with NADH, oxamate, and AXKO-0046 revealed that AXKO-0046 was an allosteric inhibitor distant from the catalytic site. The binding mode of AXKO-0046 spatially correlated with SAR studies to rationalise critical functional groups. The indole ring occupied a shallow pocket between the dimer interface, and its $\mathrm{NH}$ group formed a hydrogen bond with the main chain oxygen of Ser203. The reduced LDHB inhibitory activity of the N-Me derivative (AXKO-0067) was attributed to the disappearance of the interaction. In addition, two amino groups of AXKO-0046 could form hydrogen bonds with Glu214 at the symmetry-related location of the tetramer. The methylated derivatives of AXKO-0046 (AXKO0060, AXKO-0077, and AXKO-0058) also showed reduced LDHB inhibitory activity, which may be related to the attenuation of the interactions. The glutamic acid of LDHB (Glu214) was replaced by threonine in LDHA (Supplementary Figures S8 and S9). Near the AXKO-0046 binding site, two lysine residues of LDHB (Lys308 and Lys310) were also substituted by threonine in LDHA. Thus, the selectivity of AXKO-0046 may be attributed to these amino acid differences.

There are two binding sites in the tetramer that are located at the interface of two dimers. In bacterial LDH enzymes, the allosteric site of the activator, fructose 1,6-bisphosphate (FBP), is involved in the regulation of enzymatic activity ${ }^{39}$. The FBP-binding site is also located at the dimer interface but is structurally distinct from the binding site of AXKO-0046. Moreover, the regulatory mechanism of bacterial LDHs is not applicable to the inhibitory mechanism of AXKO-0046 against LDHA and LDHB. Based on the crystal structures, the allosteric transition by the activator within two subunits is not required for the LDHB activity. Machilin A (MA) has been reported to bind to LDHA in an interfacial allosteric site close to the FBP binding site ${ }^{40}$. However, its binding conformation of MA remains controversial because steric clashes of the protein-ligand interactions have been observed. Recently, selective allosteric inhibitors of the human LDHA isoenzyme with submicromolar activity 


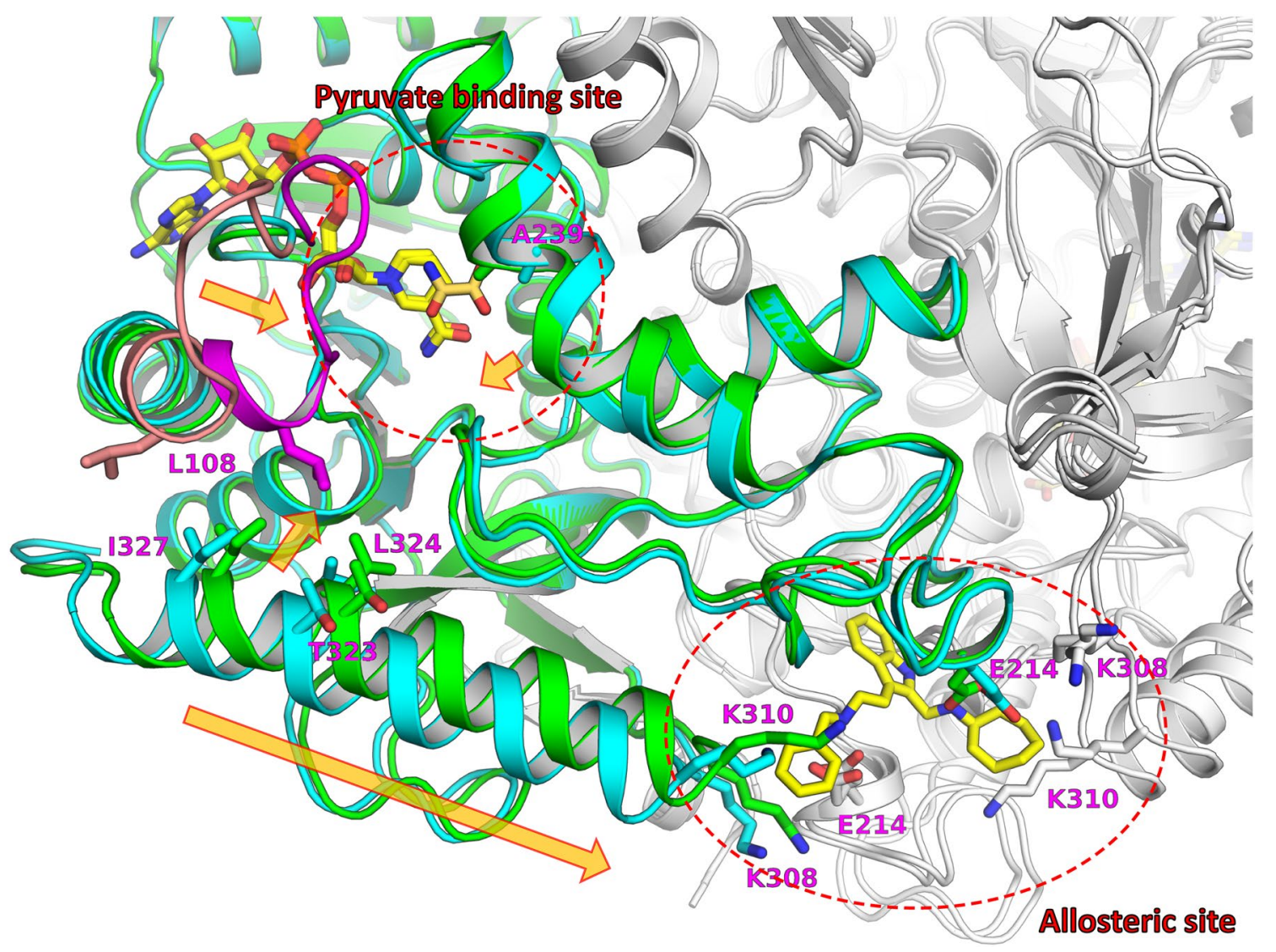

Figure 6. Superposition of the LDHB/NADH/oxamate/AXKO-0046 complex (green or magenta) with the LDHB/NADH complex (cyan or salmon). For clarity, one monomer of each tetramer is coloured, whereas others are in grey. NADH, oxamate, and AXKO-0046 are depicted as stick models in yellow, yellow-orange, and yellow, respectively. Key side chains of key residues are also depicted as stick models and labelled. The active site and allosteric site are highlighted in red dot circles. Structural rearrangements by substrate binding are indicated with orange arrows.

in vitro ${ }^{41}$. Two compounds with selectivity against LDHA are bound to a novel binding site adjacent to the active site. Overall, we concluded that the binding site of AXKO-0046 was a unique allosteric binding site of a selective LDHB inhibitor.

A structural comparison between our quaternary and binary complexes revealed that the active-site loop, referred to as the substrate-specificity loop, exhibited closed and open conformations during the binding and release of substrates, respectively (Supplementary Fig. 7). The active-site loop of the closed conformation interacted with the C-terminal helix through hydrophobic interactions among Leu108, Thr323, Leu324, and Ile327. The substantial conformational changes in the active-site loop, accompanied by the conformational shift in the C-terminal helix, were associated with the binding site of AXKO-0046 (Fig. 6). These results suggested that substrate binding mediated the forging of the allosteric binding site and facilitated the specific binding of AXKO-0046. Consequently, the AXKO-0046 binding interfered with the machinery of the enzyme, although the substrate was bound. This structural observation was consistent with the results of substrate-competition assay suggesting that the inhibitory mechanism of AXKO-0046 was uncompetitive.

In conclusion, the LDHB assay we developed to monitor LDHB activity had numerous advantages over current assay methods. In particular, our method was continuous, nonradioactive, irreversible, robust, and versatile. In this study, we showed that AXKO-0046 acted as a potent specific inhibitor of LDHB in an uncompetitive manner. Thus, AXKO-0046 may be helpful for the exploration of molecular probes to elucidate the biological functions and therapeutic relevance of $\mathrm{LDHB}$ as a drug target. The compound may also be a valuable tool to elucidate cancer metabolism. However, AXKO-0046 exhibited no potent cellular activity with clear pharmacodynamic properties. Furthermore, it may be necessary to perform lead optimisation for this series to enable in vivo evaluation at exposure levels that are not generally toxic. Our findings lay a foundation for the development of drugs to treat LDHB-related diseases.

\section{Methods}

Materials. Recombinant human LDHA and LDHB were purchased from R\&D Systems (Minneapolis, MN, USA). NAD ${ }^{+}$was purchased from MP Biomedicals (Santa Ana, CA, USA). DTT, NaCl, and Triton X-100 were obtained from Sigma-Aldrich (St. Louis, MO, USA). Dihexylammonium acetate (DHAA) was obtained from 
Tokyo Kasei Kogyo (Tokyo, Japan). NADH, pyruvate, bovine serum albumin (BSA), and other reagents were purchased from Fujifilm Wako (Osaka, Japan).

AXKO-0046 was synthesised by Takeda Pharmaceutical Co., Ltd. (Osaka, Japan); AXKO-0058, AXKO-0060, AXKO-0067, and AXKO-0077 were synthesised by Axcelead Drug Discovery Partners, Inc. (Kanagawa, Japan).

LDHB and LDHA assays for primary screening. We screened for compounds (final concentration: $10 \mu \mathrm{M}$ ) within pooled libraries consisting of more than 345,000 compounds. Each compound in DMSO (1 mM) was dispensed into 384-well V base plates (100 nL/well) using an Echo 555 acoustic dispenser (Labcyte, Sunnyvale, CA, USA). LDHB ( $5 \mu \mathrm{L})$ was diluted in assay buffer $(20 \mathrm{mM}$ Tris [pH 7.5], containing $2 \mathrm{mM} \mathrm{DTT}, 0.005 \%$ Triton X100, and $0.005 \%$ BSA) and dispensed into assay plates (final concentration: $0.25 \mathrm{nM}$ ). The plates were centrifuged $(200 \times g$ for $1 \mathrm{~min})$ and incubated at room temperature for $30 \mathrm{~min}$, and $5 \mu \mathrm{L}$ of NADH and pyruvate were added to the assay buffer (final concentration: $75 \mu \mathrm{M}$ ). After incubation for $30 \mathrm{~min}$ at room temperature, acetonitrile $(10 \mu \mathrm{L})$ and water $(50 \mu \mathrm{L})$ were added to quench the reaction. All additions were performed using a Multidrop Combi (Thermo Fisher Scientific, Waltham, MA, USA). Assay plates were sealed and centrifuged $\left(2700 \times g\right.$ for $10 \mathrm{~min}$ ) before storage at $-20^{\circ} \mathrm{C}$.

The conditions for LDHA assays were as described above, except for substrate concentrations, that is, $400 \mu \mathrm{M}$ pyruvate and $100 \mu \mathrm{M}$ NADH. These concentrations were chosen based on fitting to the Michaelis-Menten model with the $\mathrm{K}_{\mathrm{m}}$ values (Supplementary Figure S2).

High-throughput mass spectrometry. For RF-MS (Agilent, Wakefield, MA, USA), the enzyme reaction solution $(5 \mu \mathrm{L})$ was aspirated directly from the quenched assay plates and loaded onto a C8 solid-phase extraction cartridge (Agilent) with a mobile phase of water containing DHAA (5 mM) for $2500 \mathrm{~ms}$ at a flow rate of $1.25 \mathrm{~mL} / \mathrm{min}$. The analytes were then co-eluted into the mass spectrometer using water:acetonitrile:acetone $(2: 1: 1)$ containing ammonium acetate $(5 \mathrm{mM})$ for $5000 \mathrm{~ms}$ at a flow rate of $1.0 \mathrm{ml} / \mathrm{min}$. NADH and NAD ${ }^{+}$were detected by multiple reaction monitoring with QI/Q3 transitions at $m / z 664.2$ to 397.0 and $m / z 662.1$ to 540.1 , respectively, on a Sciex API4000 triple quadrupole mass spectrometer (Applied Biosystems, Foster City, CA, USA) in the negative electrospray ionization mode. The extracted ion chromatograms for each transition were integrated and processed using RapidFire Integrator (Agilent). The data for each well were normalised using the monitoring product conversion with the ratio of $\mathrm{AUC}_{\text {product }} /\left(\mathrm{AUC}_{\text {product }}+\mathrm{AUC}_{\text {substrate }}\right)$.

To determine the compound activities, percent inhibition data normalised to $0 \%$ (DMSO only) and $100 \%$ inhibition (no-enzyme control) wells were calculated. Data analysis was performed using the Tibco Spotfire (Boston, MA, USA) packages.

Substrate-competition assay. To evaluate the mechanism of inhibition of the selected compounds, their initial reaction velocities were measured using five concentrations of NADH or pyruvate $(10,30,50,100$, and $200 \mu \mathrm{M})$ incubated with $100 \mu \mathrm{M}$ pyruvate for NADH titration or $100 \mu \mathrm{M}$ NADH for pyruvate titration. A solution of AXKO-0046 $(0.00001,0.0001,0.001,0.01,0.1,1,10$, and $100 \mu \mathrm{M})$ with $0.25 \mathrm{nM}$ LDHB was then added. The enzymatic reactions were performed at room temperature for $15 \mathrm{~min}$ in triplicate. At each inhibitor concentration, the dependence of the initial reaction velocity on the substrate concentration was fitted to a nonlinear Michaelis-Menten model to obtain the $\mathrm{K}_{\mathrm{m}}$ and $\mathrm{V}_{\max }$ values using GraphPad Prism. Lineweaver-Burk plots were generated by superimposing the data of $1 /$ [velocity] and $1 /$ [substrate] and the line corresponding to the Michaelis-Menten nonlinear fit.

Protein preparation for structural analysis. Human LDHB (Ala2-Leu334; NCBI Reference Sequence: NM_001174097.2) DNA was synthesised and ligated into a pET21a vector (Merck Millipore, Darmstadt, Germany) with an N-terminal His-Avi tag followed by a TEV protease cleavage site using VectorBuilder (Chicago, IL, USA). The expression plasmid was transfected into Escherichia coli BL21 (DE3) (Nippon Gene, Toyama, Japan), and the cells were grown in lysogeny broth medium containing ampicillin $(100 \mathrm{mg} / \mathrm{mL})$. Protein expression was induced with isopropyl $\beta$-D-1-thiogalactopyranoside $(0.2 \mathrm{mM})$ following culture for $16 \mathrm{~h}$ at $16^{\circ} \mathrm{C}$. The harvested cells were lysed by sonication in lysis buffer ( $50 \mathrm{mM}$ Tris, $\mathrm{pH}$ 8.0) containing $\mathrm{NaCl}(150 \mathrm{mM})$ nuclease $(5 \mathrm{U} / \mathrm{mL})$ and centrifuged at $15,000 \times g$ for $10 \mathrm{~min}$ at $4{ }^{\circ} \mathrm{C}$. The clarified supernatant was loaded onto an Ni-NTA cartridge (FUJIFILM Wako, Osaka, Japan), and the eluted fraction was purified on a HiLoad 26/60 Superdex 200 pg column (GE Healthcare, Piscataway, NJ, USA). The His-Avi tag was digested using TEV protease (SigmaAldrich), and the digested solution was subsequently passed through an Ni-NTA column to remove TEV protease and the uncleaved protein.

The protein was further purified by anion-exchange chromatography (monoQ; GE Healthcare) with a linear gradient of $\mathrm{NaCl}$. The products of all purification steps were assessed by sodium dodecyl sulfate polyacrylamide gel electrophoresis and Coomassie Blue staining (Sigma-Aldrich). The final yield of protein was $46 \mathrm{mg}$ for every $1 \mathrm{~L}$ of culture. For crystallisation, the purified protein was buffer-exchanged to the final buffer $(50 \mathrm{mM}$ Tris [pH 7.6] with $150 \mathrm{mM} \mathrm{NaCl})$, concentrated $(20 \mathrm{mg} / \mathrm{mL}$ ) by ultrafiltration (AMICON-ULTRA $10 \mathrm{~K}$; Millipore, Bedford, MA, USA), and stored at $-80^{\circ} \mathrm{C}$.

X-ray crystallography. The complexes of LDHB with NADH or NADH, oxamate, and AXKO-0046 were generated by incubating threefold molar excesses of ligands on ice for 2-3 h before crystallisation experiments. Both complexes were crystallised from a reservoir solution containing HEPES (0.1 M [pH 7.5]), potassium formate or ammonium acetate $(0.2 \mathrm{M})$, and PEG $3350(20 \% \mathrm{v} / \mathrm{v})$ at $20^{\circ} \mathrm{C}$ via the sitting-drop vapour diffusion method. Prior to data collection, crystals were immersed in the reservoir solution containing ethylene glycol $(30 \%)$ as a cryoprotectant and flash-frozen in liquid nitrogen. Diffraction data were collected from a single 
crystal using a DECTRIS Pilatus3-S6M PAD detector (Baden-Daettwil, Switzerland) with a BL45XU beamline (SPring-8, Hyogo, Japan) under a 100-K nitrogen cryostream. The diffraction data were reduced and scaled using HKL2000 ${ }^{42}$.

The structure was solved following the molecular replacement method using Phaser ${ }^{43}$ in the CCP4 software suite ${ }^{44}$ and the LDHB structure (PDB code $1 \mathrm{I}^{2} \mathrm{Z}^{18}$ ) as a search model. Refinement was performed using REFMAC $5^{45}$ with individual isotropic restrained B factors. Some data (5\%) were set aside for crossvalidation before refinement, and progress was monitored using $R_{\text {free. }}$. For TLS refinement, the tetramer of the protein and ligands was set as a single rigid body ${ }^{46}$. Interactive model building was performed using COOT $^{47}$. The final models were validated using Molprobity ${ }^{48}$. All graphical figures were generated using PyMOL (Schrödinger LLC, Cambridge, MA, USA). Crystallographic processing and refinement statistics are summarised in Supplementary Table S1.

Data analysis. For the kinetic parameters, the initial rates of $\mathrm{LDH}$ activity were determined by incubating LDHA or LDHB with various concentrations of NADH and pyruvate in the assay buffer at room temperature. The reaction product was measured by RF-MS. To estimate the $\mathrm{K}_{\mathrm{m}}$ and $\mathrm{V}_{\max }$ values, the initial rates were fit to the Michaelis-Menten equation. $\mathrm{EC}_{50}$ and Hill slope calculation and curve fitting using four-parameter fits were performed using GraphPad Prism v6.07 (GraphPad Software, San Diego, CA, USA) or XLfit (IDBS, Guildford, $\mathrm{UK}$ ) with nonlinear regression analysis, wherein the $\mathrm{EC}_{50}$ value equaled the concentration at which the inflection point of the fitted model was reached. The quality and robustness of the screening campaign process were determined by analysing the Z' factor.

\section{Data availability}

The atomic coordinates and structure factors have been deposited in the Protein Data Bank with the primary accession codes 7DBJ and 7DBK. The data that support the findings of this study are available from the corresponding authors upon reasonable request.

Received: 16 July 2021; Accepted: 18 October 2021

Published online: 01 November 2021

\section{References}

1. Hsu, P. P. \& Sabatini, D. M. Cancer cell metabolism: Warburg and beyond. Cell 134, 703-707 (2008).

2. Warburg, O. On the origin of cancer cells. Science 123, 309-314 (1956).

3. Vander Heiden, M. G., Cantley, L. C. \& Thompson, C. B. Understanding the Warburg effect: The metabolic requirements of cell proliferation. Science 324, 1029-1033 (2009).

4. Valvona, C. J., Fillmore, H. L., Nunn, P. B. \& Pilkington, G. J. The regulation and function of lactate dehydrogenase A: Therapeutic potential in brain tumor. Brain Pathol. 26, 3-17 (2016).

5. Kolev, Y., Uetake, H., Takagi, Y. \& Sugihara, K. Lactate dehydrogenase-5 (LDH-5) expression in human gastric cancer: Association with hypoxia-inducible factor (HIF-lalpha) pathway, angiogenic factors production and poor prognosis. Ann. Surg. Oncol. 15, 2336-2344 (2008).

6. Koukourakis, M. I. et al. Lactate dehydrogenase-5 (LDH-5) overexpression in non-small-cell lung cancer tissues is linked to tumour hypoxia, angiogenic factor production and poor prognosis. Br. J. Cancer 89, 877-885 (2003).

7. Le, A. et al. Inhibition of lactate dehydrogenase A induces oxidative stress and inhibits tumor progression. Proc. Natl. Acad. Sci. USA 107, 2037-2042 (2010).

8. Sheng, S. L. et al. Knockdown of lactate dehydrogenase A suppresses tumor growth and metastasis of human hepatocellular carcinoma. FEBS J. 279, 3898-3910 (2012).

9. Billiard, J. et al. Quinoline 3-sulfonamides inhibit lactate dehydrogenase A and reverse aerobic glycolysis in cancer cells. Cancer Metab. 1, 19 (2013).

10. McCleland, M. L. et al. Lactate dehydrogenase B is required for the growth of KRAS-dependent lung adenocarcinomas. Clin. Cancer Res. 19, 773-784 (2013).

11. McCleland, M. L. et al. An integrated genomic screen identifies LDHB as an essential gene for triple-negative breast cancer. Cancer Res. 72, 5812-5823 (2012).

12. Satoh, K. et al. Global metabolic reprogramming of colorectal cancer occurs at adenoma stage and is induced by MYC. Proc. Natl. Acad. Sci. USA 114, E7697-E7706 (2017).

13. Brisson, L. et al. Lactate dehydrogenase B controls lysosome activity and autophagy in cancer. Cancer Cell 30, 418-431 (2016).

14. White, E. Deconvoluting the context-dependent role for autophagy in cancer. Nat. Rev. Cancer 12, 401-410 (2012).

15. LeVan, K. M. \& Goldberg, E. Properties of human testis-specific lactate dehydrogenase expressed from Escherichia coli. Biochem. J. 273, 587-592 (1991).

16. Matoba, Y. et al. An alternative allosteric regulation mechanism of an acidophilic l-lactate dehydrogenase from Enterococcus mundtii 15-1A. FEBS Open Bio 4, 834-847 (2014).

17. Choi, S.-R. et al. Generation of oxamic acid libraries: Antimalarials and inhibitors of Plasmodium falciparum lactate dehydrogenase. J. Comb. Chem. 9, 292-300 (2007).

18. Choi, S.-R. et al. Design, synthesis, and biological evaluation of Plasmodium falciparum lactate dehydrogenase inhibitors. J. Med. Chem. 50, 3841-3850 (2007).

19. Pirrung, M. C. et al. Methyl scanning: Total synthesis of demethylasterriquinone B1 and derivatives for identification of sites of interaction with and isolation of its receptor(s). J. Am. Chem. Soc. 127, 4609-4624 (2005).

20. Read, J. A., Winter, V. J., Eszes, C. M., Sessions, R. B. \& Brady, R. L. Structural basis for altered activity of M- and H-isozyme forms of human lactate dehydrogenase. Proteins 43, 175-185 (2001).

21. Dempster, S., Harper, S., Moses, J. E. \& Dreveny, I. Structural characterization of the apo form and NADH binary complex of human lactate dehydrogenase. Acta Crystallogr. D Biol. Crystallogr. 70, 1484-1490 (2014).

22. Kolappan, S. et al. Structures of lactate dehydrogenase A (LDHA) in apo, ternary and inhibitor-bound forms. Acta Crystallogr. D Biol. Crystallogr. 71, 185-195 (2015).

23. Thabault, L. et al. Interrogating the lactate dehydrogenase tetramerization site using (stapled) peptides. J. Med. Chem. 63, 4628-4643 (2020).

24. Kim, S. Y. Cancer energy metabolism: Shutting power off cancer factory. Biomol. Ther. 26, 39-44 (2018). 
25. Yoshida, G. J. Metabolic reprogramming: The emerging concept and associated therapeutic strategies. J. Exp. Clin. Cancer Res. 34, 111 (2015).

26. Sonveaux, P. et al. Targeting lactate-fueled respiration selectively kills hypoxic tumor cells in mice. J Clin Invest. 118, 3930-3942 (2008).

27. Leite, J. S., Martins, S. C., Oliveira, J., Cunha, M. F. \& Castro-Sousa, F. Clinical significance of macroscopic completeness of mesorectal resection in rectal cancer. Colorectal Dis. 13, 381-386 (2011).

28. Beloueche-Babari, M. et al. Monocarboxylate transporter 1 blockade with AZD3965 inhibits lipid biosynthesis and increases tumour immune cell infiltration. Br. J. Cancer 122, 895-903 (2020).

29. Okumura, N. et al. Genetic analyses in homozygous and heterozygous variants of lactate dehydrogenase-B (H) subunit-LD-B Matsumoto I and II (LD-B W323R). Clin. Chim. Acta 287, 163-171 (1999).

30. Sudo, K. et al. A novel in-frame deletion mutation in a case of lactate dehydrogenase (LD) $H$ subunit deficiency showing an atypical LD isoenzyme pattern in serum and erythrocytes. Clin. Biochem. 32, 137-141 (1999).

31. Yoshikuni, K., Tagami, H., Yamada, M., Sudo, K. \& Kanno, T. Erythematosquamous skin lesions in hereditary lactate dehydrogenase M-subunit deficiency. Arch. Dermatol. 122, 1420-1424 (1986).

32. Kanno, T. et al. Lactate dehydrogenase M-subunit deficiency: A new type of hereditary exertional myopathy. Clin. Chim. Acta 173, 89-98 (1988).

33. Granchi, C. et al. Discovery of N-hydroxyindole-based inhibitors of human lactate dehydrogenase isoform A (LDH-A) as starvation agents against cancer cells. J. Med. Chem. 54, 1599-1612 (2011).

34. Vanderporten, E. et al. Label-free high-throughput assays to screen and characterize novel lactate dehydrogenase inhibitors. Anal. Biochem. 441, 115-122 (2013).

35. Zhai, X., Yang, Y., Wan, J., Zhu, R. \& Wu, Y. Inhibition of LDH-A by oxamate induces G2/M arrest, apoptosis and increases radiosensitivity in nasopharyngeal carcinoma cells. Oncol. Rep. 30, 2983-2991 (2013).

36. Manerba, M. et al. Galloflavin (CAS 568-80-9): A novel inhibitor of lactate dehydrogenase. ChemMedChem 7, 311-317 (2012).

37. Ward, R. A. et al. Design and synthesis of novel lactate dehydrogenase A inhibitors by fragment-based lead generation. J. Med. Chem. 55, 3285-3306 (2012).

38. Yu, Q. \& Heikal, A. A. Two-photon autofluorescence dynamics imaging reveals sensitivity of intracellular NADH concentration and conformation to cell physiology at the single-cell level. J. Photochem. Photobiol. B Biol. 95, 46-57 (2009).

39. Iwata, S., Kamata, K., Yoshida, S., Minowa, T. \& Ohta, T. T and R states in the crystals of bacterial L-lactate dehydrogenase reveal the mechanism for allosteric control. Nat. Struct. Biol. 1, 176-185 (1994).

40. Chung, T. W. et al. Machilin A inhibits tumor growth and macrophage M2 polarization through the reduction of lactic acid. Cancers 11, 963 (2019)

41. Anders Friberg, A. et al. Structural evidence for isoform-selective allosteric inhibition of lactate dehydrogenase A. ACS Omega 27, 13034-13041 (2020).

42. Otwinowski, Z. \& Minor, W. Processing of X-ray diffraction data collected in oscillation mode. Methods Enzymol. 276, 307-326 (1997).

43. McCoy, A. J. et al. Phaser crystallographic software. J. Appl. Crystallogr. 40, 658-674 (2007).

44. Winn, M. D. et al. Overview of the CCP4 suite and current developments. Acta Crystallogr. D Biol. Crystallogr. 67, 235-242 (2011).

45. Murshudov, G. N., Vagin, A. A. \& Dodson, E. J. Refinement of macromolecular structures by the maximum-likelihood method. Acta Crystallogr. D Biol. Crystallogr. 53, 240-255 (1997).

46. Winn, M. D., Isupov, M. N. \& Murshudov, G. N. Use of TLS parameters to model anisotropic displacements in macromolecular refinement. Acta Crystallogr. D Biol. Crystallogr. 57, 122-133 (2001).

47. Emsley, P., Lohkamp, B., Scott, W. G. \& Cowtan, K. Features and development of coot. Acta Crystallogr. D Biol. Crystallogr. 66, 486-501 (2010).

48. Chen, V. B. et al. MolProbity: All-atom structure validation for macromolecular crystallography. Acta Crystallogr. D Biol. Crystal$\log$. 66, 12-21 (2010).

\section{Acknowledgements}

We thank the following colleagues at the Axcelead Drug Discovery Partners for their valuable support: Ms. Mai Okada for evaluation of the compounds and Mr. Takamitsu Maru and Mr. Hideyuki Oki for assistance with biophysical assays. We also thank Dr. Junji Matsui for supervision of the study and valuable suggestions.

\section{Author contributions}

T.S. conceptualised and coordinated the study. A.N., S.K., and T.K. conceived and designed the experiments. S. Shibata screened the compound libraries, performed inhibition studies, and wrote the manuscript. S. Sogabe performed protein crystallization, analysed the diffraction data, and wrote the manuscript. N.T. and T.F. designed and synthesised the chemical compound. All authors critically revised the content and approved the final version for submission.

\section{Competing interests}

The authors declare no competing interests.

\section{Additional information}

Supplementary Information The online version contains supplementary material available at https://doi.org/ 10.1038/s41598-021-00820-7.

Correspondence and requests for materials should be addressed to S.S. or T.K.

Reprints and permissions information is available at www.nature.com/reprints.

Publisher's note Springer Nature remains neutral with regard to jurisdictional claims in published maps and institutional affiliations. 
(c) (i) Open Access This article is licensed under a Creative Commons Attribution 4.0 International cc) License, which permits use, sharing, adaptation, distribution and reproduction in any medium or format, as long as you give appropriate credit to the original author(s) and the source, provide a link to the Creative Commons licence, and indicate if changes were made. The images or other third party material in this article are included in the article's Creative Commons licence, unless indicated otherwise in a credit line to the material. If material is not included in the article's Creative Commons licence and your intended use is not permitted by statutory regulation or exceeds the permitted use, you will need to obtain permission directly from the copyright holder. To view a copy of this licence, visit http://creativecommons.org/licenses/by/4.0/.

(C) The Author(s) 2021 